Review Article

\title{
Clinimetrics of the 9- and 19-Item Wearing-Off Questionnaire: A Systematic Review
}

\author{
Carlos E. Mantese $\mathbb{D}^{1},{ }^{1,2}$ Artur Schumacher-Schuh, ${ }^{1}$ and Carlos R. M. Rieder $\mathbb{D}^{1,3,4}$ \\ ${ }^{1}$ Postgraduate Program in Medical Sciences, Universidade Federal do Rio Grande do Sul, Porto Alegre, RS, Brazil \\ ${ }^{2}$ Mãe de Deus Hospital, Porto Alegre, RS, Brazil \\ ${ }^{3}$ Universidade Federal de Ciências da Saúde de Porto Alegre (UFCSPA), Porto Alegre, RS, Brazil \\ ${ }^{4}$ Hospital de Clínicas de Porto Alegre (HCPA), Porto Alegre, RS, Brazil \\ Correspondence should be addressed to Carlos R. M. Rieder; carlosrieder@gmail.com
}

Received 8 October 2017; Revised 17 January 2018; Accepted 19 February 2018; Published 1 April 2018

Academic Editor: Peter Hagell

Copyright $\odot 2018$ Carlos E. Mantese et al. This is an open access article distributed under the Creative Commons Attribution License, which permits unrestricted use, distribution, and reproduction in any medium, provided the original work is properly cited.

\begin{abstract}
The treatment of Parkinson's disease (PD) with dopaminergic therapy improves functionality and quality of life. However, as the disease progresses, the wearing-off phenomenon develops, which necessitates complex posology adjustment or adjuvant therapy. This phenomenon may not be well recognized, especially if it is mild or involves nonmotor symptoms. Questionnaires were developed to improve the recognition of the wearing-off phenomenon. The questionnaires consist of a list of symptoms that patients must check if they have and if the symptoms improve with medication. A recent review by the Movement Disorder Society suggested the 19-item (WOQ-19) and 9-item (WOQ-9) questionnaires as screening tools for the wearing-off phenomenon. However, there has not been a systematic review to assess the questionnaires' clinimetric properties, such as sensitivity, specificity, test-retest reliability, and responsiveness. We conducted an extensive search for studies using these two tools. We identified 3 studies using WOQ-19 and 5 studies using WOQ-9. Both questionnaires seem to have good sensitivity (0.81-1). WOQ19 has variable specificity (0.39-0.8), depending on the number of positive items, while WOQ-9 lacks specificity (0.1-0.69). Only one study using WOQ-19 reported test-retest, and only two studies reported responsiveness. Thus, this report describes the first independent systematic review to exam quantitatively the clinimetric properties of these two questionnaires.
\end{abstract}

\section{Introduction}

The treatment of Parkinson's disease (PD) with dopaminergic therapy improves functionality and quality of life. However, as the disease progresses, it causes motor and nonmotor fluctuations [1]. The well-described wearing-off (WO) phenomenon is the shortening effect of levodopa, which can be managed with dosage adjustment or adjuvant therapy, such as catechol-O-methyltransferase (COMT) inhibitors [2]. Clinical evaluation has been the gold standard for diagnosing this condition. However, the WO phenomenon may not be well recognized, mainly if it is mild or involves nonmotor symptoms. Several scholars argue that recognition of WO phenomenon could change the way that it is managed and improve patient's functionality [3].
To improve the recognition of WO phenomenon, a 32-item questionnaire (WOQ-32) was developed [3]. The questionnaire consists of a checklist of symptoms that patients must identify, and they must note if these symptoms improve with medication. For practical reasons, using the same research, this questionnaire was adapted to a 19-item questionnaire (WOQ-19), which had the same properties [4]. Later, a 9-item questionnaire (WOQ-9) was developed [5], containing the most valuable questions, and it was successfully tested [6]. The WOQ-9 has been used for a number of clinical studies, translated into several languages, and adapted with several different clinimetric properties [7]. A recent review by the Movement Disorder Society set both the WOQ-19 and WOQ-9 as recommended tools for screening for WO phenomenon [8]. However, this 


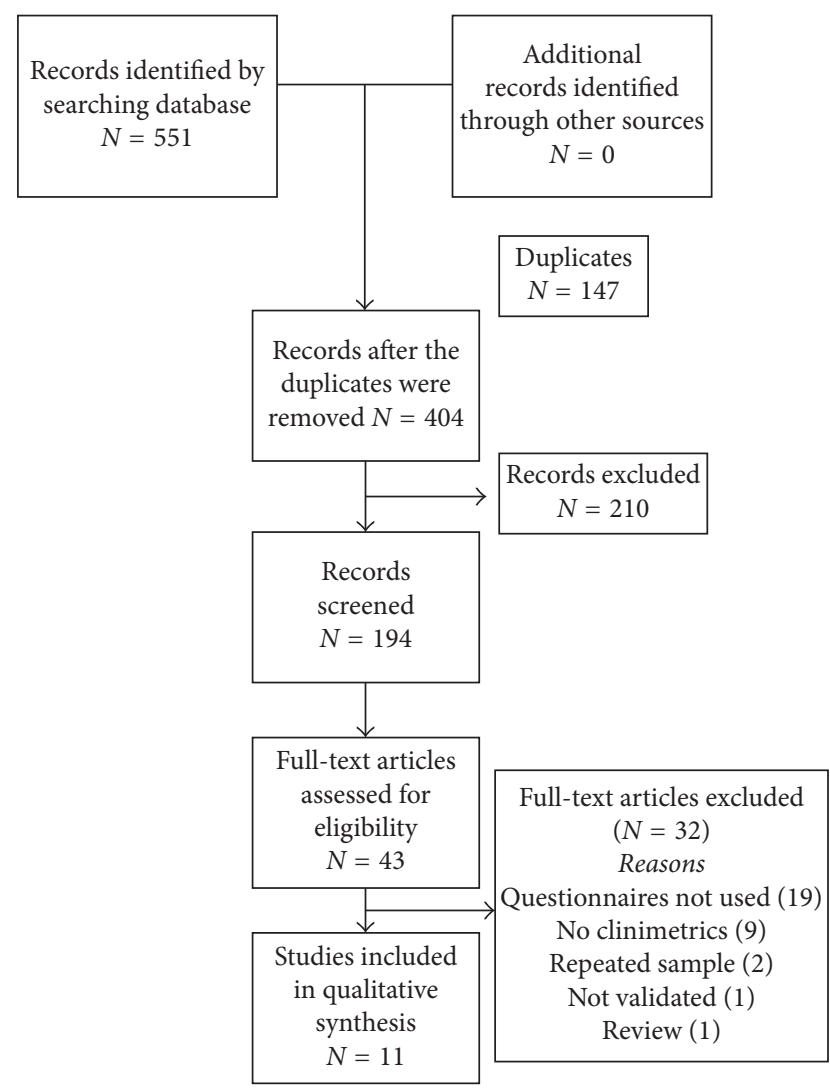

FIgURE 1: Flow diagram for data extraction.

review did not address quantitatively the clinimetric properties in Parkinson's disease patients compared to clinical evaluation.

Thus, we conducted a systematic review and analysis of the clinimetric properties of both the WOQ-19 and WOQ-9 questionnaires, such as sensitivity, specificity, predictive positive value (PPV), negative predictive value (NPV), and stability with test-retest and responsiveness.

\section{Methods}

We follow the PRISMA statement.

The inclusion criterion was studies using WOQ-9 or WOQ-19 in PD patients to diagnose WO compared to the gold-standard, clinical evaluation. The studies must examine sensitivity and specificity, or they must include data that we could calculate. Also, we include studies using one of the questionnaires if they employed data regarding test-retest or responsiveness. Formal validation was not required [9], but at least a translation and face validation for the given language was applied. Reviews, abstracts, and conference meetings were excluded. Responsiveness was calculated following Cohen's effect size [10].

The search was conducted in MEDLINE, Embase, and Web of Sciences between 01/06/2017 and 22/12/17. The terms were ((Parkinson's OR Parkinson's disease) AND (wearing off OR wearing-off OR motor fluctuation) AND (questionnaire)). There was also a bibliography review for the select articles and reviews already published. The articles were independently selected by title for abstract reading by two reviewers (Artur Schumacher-Schuh and Carlos E. Mantese). In case of disagreement, the articles were discussed by another author (Carlos R. M. Rieder). Later, a number of articles were selected for full reading based on abstract information.

\section{Results}

As shown in Figure 1, we observed 404 articles based on the title after excluding duplicates. We reviewed 194 abstracts and 43 full-text articles after excluding 4 abstracts. Ultimately, we selected 3 articles about WOQ-19 [11-13] and 5 articles about WOQ-9 [6, 14-17] for sensitivity and specificity, 1 for WOQ-19 test-retest [18], 2 for responsiveness, 1 for WOQ-9 [19], and 1 for WOQ-19 [20]. Of note, we did not include the original WOQ-19 description [4] because it was an adaptation of WOQ-32, as was WOQ-9 [5]. In addition to these articles, we exclude 17 more because they did not use the target questionnaires; among these 17, two more were from the same 32-item questionnaire $[3,21]$ and 15 from other means of assessment [22-36]. One article was not used because it lacked any type of language validation [37], and two articles presented post hoc analyses of the same populations outlined in other articles that we include [38, 39]. Nine articles did not present clinimetric properties [40-48], and among them, two provided data using which we could calculate only PPV [41, 43]. One was a review [7]. One paper [18] included for test-retest was not included for sensitivity and specificity because it used the same population as another article [13].

We did not identify any articles that were not included in the search from original libraries.

In WOQ-19, there are 3 trials selected (Table 1). One of them used 1-item cutoff [12], while the others [11, 13] used 2 -item cutoff. The sensitivity ranged from 0.81 to 0.90 , and the specificity was $0.39-0.80$. PPV was $0.62-0.88$, and NPV was $0.64-0.84$. The wide range of specificity seems secondary to one study that used 1-item cutoff. This trial does not exhibit better aggregate sensitivity but has shown worse specificity.

In WOQ-9 (Table 2), all studies had 1-item cutoff. Sensibility was $0.87-1$, specificity was $0.10-0.69$, PPV was $0.48-0.86$, and NPV was $0.71-1$. All studies showed excellent sensitivity but lacked specificity.

Test-retest stability was assessed in one paper [18], two weeks apart from each test, which showed an intraclass correlation of number of positive items of 0.858 . It was applied to stable patients; however, it did not mention the clinical stability or the type of intraclass correlation.

For responsiveness, two studies were analyzed $[19,20]$. Both were clinical trials with COMT inhibitors. One of them [19] used WOQ-9 and reported improvement in most items in proportion of patients with improvements; however, it did not provide data to calculate the effect size. In the other trial [20], Cohen's effect size was 0.5.

\section{Discussion}

This report describes the first systematic review of quantitative clinimetric properties of WOQ-19 and WOQ-9. 
TABle 1: WOQ-19.

\begin{tabular}{lcccccc}
\hline Author & Year & $N$ & Cutoff & Sn & Sp & PPV \\
\hline Martinez-Martin et al. [11] & 2008 & 222 & 2 & 0.88 & 0.8 & 0.88 \\
Seki et al. [12] & 2013 & 464 & 1 & 0.81 & 0.39 & 0.62 \\
Stocchi et al. [13] & 2014 & 617 & 2 & 0.90 & 0.63 & 0.79 \\
\hline
\end{tabular}

Sn: sensitivity; Sp: specificity; PPV: positive predictive value; NPV: negative predictive value.

TABle 2: WOQ-9.

\begin{tabular}{lccccccc}
\hline Author & Year & $N$ & Cut-off & Sn & Sp & PPV & NPV \\
\hline Stacy et al. [6] & 2008 & 216 & 1 & 0.96 & 0.40 & 0.48 & 0.94 \\
Chan et al. [14] & 2011 & 101 & 1 & 0.87 & 0.69 & 0.86 & 0.71 \\
Bares et al. [15] & 2012 & 563 & 1 & 0.98 & 0.27 & 0.72 & 0.91 \\
Santos et al. [16] & 2014 & 60 & 1 & 1 & 0.10 & 0.54 & 1 \\
Fukae et al. [17] & 2015 & 180 & 1 & 0.94 & 0.39 & 0.66 & 0.83 \\
\hline
\end{tabular}

Sn: sensitivity; Sp: specificity; PPV: positive predictive value; NPV: negative predictive value.

Systematic reviews are fundamental to summarize important data for research and clinical practices. Additionally, this report describes the first independent review of the clinimetric properties of these questionnaires.

The WOQ-19 seems to have good accuracy, which is an excellent tool in both research and clinical practice, when a 2-item cutoff is used. However, most of the trials used WOQ-9, which has excellent sensitivity but poor specificity. Thus, the WOQ-9 could be used as a screening tool to identify certain at-risk individuals, but it would need a clinical evaluation to confirm the diagnosis, as several trials have done [42, 46]. Stacy [7] has argued that office visits could fail to recognize WO, and its position as the gold standard of care may need reevaluation [6]. This hypothesis seems difficult to prove. Moreover, most clinical trials for Parkinson's disease treatment use wearing-off outcomes diaries or UPDRS (Unified Parkinson's Disease Rating Scale) wearing-off subitems. Raciti et al. [49] showed that UPDRS has 0.87 sensitivity and 0.43 specificity compared to clinical evaluation, which make it similar to WOQ-9 and considerably worse than WOQ-19.

The variability in WOQ-19 can be explained by several reasons. In WOQ-19, one clinical trial used the 1-item cutoff and therefore lost specificity. In a ROC curve plotted by Martinez-Martin et al. [11], the questionnaire showed better accuracy when the 2-item cutoff was used. As in WOQ-9, the 1 -item cutoff seems to have the same lack of specificity. Fukae et al. [17] showed that when the 2-item cutoff is used, the WOQ-9's specificity improves (from 0.39 to 0.72 ) and loses a little sensitivity (from 0.94 to 0.87 ). Additionally, each study involved different languages, and the final result depended on, in part, the properties of each specific validation. Moreover, the gold standard could be different depending on the physician's expertise (i.e., if they are movement disorder specialists or in-training neurologists). Finally, while most clinical trials excluded patients who could not complete the questionnaires, certain differences in educational, cultural, and social backgrounds could explain a portion of the variability in the questionnaires.
Of note, we did not identify information regarding questionnaire reliability or validity other than criteria validity. Furthermore, only one article on WOQ-19 examined test-retest through intraclass correlation of number of positive items, and it did not mention the type, consistency, or agreement. The second is preferred [50]. For test-retest, even not mentioned clinical stability, two weeks apart from each test seems enough time in Parkinson's disease to avoid recall bias and ensure clinical stability. We have not found any paper with kappa agreement from individual question. Being a questionnaire with dichotomous responses, the use of kappa would seem appropriate. We did not identify any reports of test-retest for WOQ-9. Responsiveness was obtained from two clinical trials for WOQ-19 comparing add-on therapy with entacapone (a COMT inhibitor). This therapy is used to treat WO phenomenon, and both showed an improvement of questionnaire on the basis of number positive items. However, in one trial, we have no data to calculate the effect size [18]. The other one [19] showed effect size of 0.5 , which means a moderate effect.

The lack of data regarding reliability and even validation by means other than criteria validation might be observed because the original study used WOQ-32, and later, the WOQ-19 and WOQ-9 were developed, and even those questionnaires were not tested for those properties by the developers. Additionally, we did not include conference meetings or abstracts, which can account for the loss of certain data (even so, we did not identify those data in the libraries we searched). This information is important to clinicians and researchers because it might influence how they use questionnaires. A questionnaire with poor testretest performance is not reliable to use, and the results can change with no change in clinical status.

We excluded several important articles, such as Stacy et al.'s [3] description of the WOQ-32 questionnaire and its transformation into the WOQ-19 [4] and WOQ-9 [5]. However, this article involves data from a different questionnaire, which was later transformed into the WOQ-19 and WOQ-9 questionnaires. Several articles were post hoc analyses of primary data, which we had already included. One study did not have any type of validation and did not meet our inclusion criteria. Most of the excluded trials were with no comparator; therefore, we could not address clinimetric properties.

\section{Conclusions}

We conducted the first systematic review of WOQ-19 and WOQ-9, an important tool for screening and diagnosing WO. The lack of certain data suggests caution when using the WOQ-9. However, the WOQ-19 exhibits reliability and 
was validated to use as a diagnostic tool. Moreover, we suggest that the authors report complete properties when they are publishing papers validating their methods.

\section{Conflicts of Interest}

The authors declare that there are no conflicts of interest regarding the publication of this paper.

\section{Acknowledgments}

Carlos R. M. Rieder was supported by the Brazilian government research grant agency CNPq (no. 311970/2014-1). The authors also thank the Fundo de Incentivo à Pesquisa e Eventos (FIPE), Hospital de Clínicas de Porto Alegre (HCPA), for article processing charges.

\section{References}

[1] C. C. Aquino and S. H. Fox, "Clinical spectrum of levodopainduced complications," Movement Disorders, vol. 30, no. 1, pp. 80-89, 2015.

[2] R. Pahwa and K. E. Lyons, "Levodopa-related wearing-off in Parkinson's disease: identification and management," Current Medical Research and Opinion, vol. 25, no. 4, pp. 841-849, 2009.

[3] M. Stacy, A. Bowron, M. Guttman et al., "Identification of motor and nonmotor wearing-off in Parkinson's disease: comparison of a patient questionnaire versus a clinician assessment," Movement Disorders, vol. 20, no. 6, pp. 726-733, 2005.

[4] M. Stacy and R. Hauser, "Development of a patient questionnaire to facilitate recognition of motor and non-motor wearing-off in Parkinson's disease," Journal of Neural Transmission, vol. 114, no. 2, pp. 211-217, 2007.

[5] M. Stacy, R. Hauser, W. Oertel et al., "End-of-dose wearing off in Parkinson disease: a 9-question survey assessment," Clinical Neuropharmacology, vol. 29, no. 6, pp. 312-321, 2006.

[6] M. A. Stacy, J. M. Murphy, D. R. Greeley et al., "The sensitivity and specificity of the 9-item wearing-off questionnaire," Parkinsonism and Related Disorders, vol. 14, no. 3, pp. 205-212, 2008.

[7] M. Stacy, "The wearing-off phenomenon and the use of questionnaires to facilitate its recognition in Parkinson's disease," Journal of Neural Transmission, vol. 117, no. 7, pp. 837-846, 2010.

[8] A. Antonini, P. Martinez-Martin, R. K. Chaudhuri et al., "Wearing-off scales in Parkinson's disease: critique and recommendations," Movement Disorders, vol. 26, no. 12, pp. 2169-2175, 2011.

[9] D. E. Beaton, C. Bombardier, F. Guillemin, and M. B. Ferraz, "Guidelines for the process of cross-cultural adaptation of selfreport measures," SPINE, vol. 25, no. 24, pp. 3186-3191, 2000.

[10] G. R. Norman, K. W. Wyrwich, and D. L. Patrick, "The mathematical relationship among different forms of responsiveness coefficients," Quality of Life Research, vol. 16, no. 5, pp. 815-822, 2007.

[11] P. Martinez-Martin, E. Tolosa, B. Hernandez, and X. Badia, "Validation of the "QUICK" questionnaire-a tool for diagnosis of "Wearing-Off" in patients with Parkinson's disease," Movement Disorders, vol. 23, no. 6, pp. 830-836, 2008.

[12] M. Seki, K. Takahashi, D. Uematsu et al., "Clinical features and varieties of non-motor fluctuations in Parkinson's disease: a Japanese multicenter study," Parkinsonism and Related Disorders, vol. 19, no. 1, pp. 104-108, 2013.
[13] F. Stocchi, A. Antonini, P. Barone et al., "Early detection of wearing off in Parkinson disease: the DEEP study," Parkinsonism and Related Disorders, vol. 20, no. 2, pp. 204-211, 2014.

[14] A. Chan, Y. F. Cheung, M. Au Yeung et al., "A validation study of the Chinese wearing off questionnaire 9-symptom for Parkinson's disease," Clinical Neurology and Neurosurgery, vol. 113, no. 7, pp. 538-540, 2011.

[15] M. Bareš, I. Rektorová, R. Jech et al., "Does WOQ-9 help to recognize symptoms of non-motor wearing-off in Parkinson's disease?," Journal of Neural Transmission, vol. 119, no. 3, pp. 373-380, 2012.

[16] J. G. Santos, H. F. Chien, and E. R. Barbosa, "Specificity and sensibility of 9-items wearing-off questionnaire in Brazilian Parkinson disease patient sample," Arquivos de Neuro-Psiquiatria, vol. 72, no. 11, pp. 867-873, 2014.

[17] J. Fukae, M. Higuchi, S. Yanamoto et al., "Utility of the Japanese version of the 9-item wearing-off questionnaire," Clinical Neurology and Neurosurgery, vol. 134, pp. 110-115, 2015.

[18] G. Abbruzzese, A. Antonini, P. Barone et al., "Linguistic, psychometric validation and diagnostic ability assessment of an Italian version of a 19-item wearing-off questionnaire for wearing-off detection in Parkinson's disease," Neurological Sciences, vol. 33, no. 6, pp. 1319-1327, 2012.

[19] K. Eggert, O. Skogar, K. Amar et al., "Direct switch from levodopa/benserazide or levodopa/carbidopa to levodopa/carbidopa/entacapone in Parkinson's disease patients with wearing-off: efficacy, safety and feasibilityan open-label, 6-week study," Journal of Neural Transmission, vol. 117, no. 3, pp. 333-342, 2010.

[20] E. Tolosa, B. Hernández, G. Linazasoro et al., "Efficacy of levodopa/carbidopa/entacapone versus levodopa/carbidopa in patients with early Parkinson's disease experiencing mild wearing-off: a randomised, double-blind trial," Journal of Neural Transmission, vol. 121, no. 4, pp. 357-366, 2014.

[21] J.-P. Azulay, F. Durif, R. Rogez et al., "Étude Précoce: évaluation d'un autoquestionnaire de déspistage et prise en charge précoces des fluctuations dans la maladie de Parkinson," Revue Neurologique, vol. 164, no. 4, pp. 354-362, 2008.

[22] N. Hattori, K. Fujimoto, and T. Kondo, "Patient perspectives on Parkinson's disease therapy in Japan and the United States: results of two patient surveys," Patient Related Outcome Measure, vol. 3, pp. 31-38, 2012.

[23] P. Santens and A. M. de Noordhout, "Detection of motor and non-motor symptoms of end-of dose wearing-off in Parkinson's disease using a dedicated questionnaire: a Belgian multicenter survey," Acta Neurologica Belgica, vol. 106, no. 3, pp. 137-141, 2006.

[24] P. Martinez-Martin and B. Hernandez, "Q10 study group. The Q10 questionnaire for detection of wearing-off phenomena in Parkinson's disease," Parkinsonism Related Disorder, vol. 18, no. 4, pp. 382-385, 2012.

[25] A. Schapira, S. Fox, R. Hauser et al., "Assessment of safety and efficacy of safinamide as a levodopa adjunct in patients with Parkinson disease and motor fluctuations: a randomized clinical trial," JAMA Neurology, vol. 74, no. 2, pp. 216-224, 2017.

[26] S. Cheon, M. Park, W. Kim et al., "Non-motor off symptoms in Parkinson's disease," Journal of Korean Medical Science, vol. 24, no. 2, pp. 311-314, 2009.

[27] R. Hauser, M. Panisset, G. Abbruzzese et al., "Double-blind trial of levodopa/carbidopa/entacapone versus levodopa/carbidopa in early Parkinson's disease," Movement Disorders, vol. 24, no. 4, pp. 541-550, 2009.

[28] A. Boiko, T. Batysheva, N. Minaeva et al., "Use of the new levodopa agent Stalevo (levodopa/carbidopa/entacapone) in 
the treatment of Parkinson's disease in out-patient clinical practice (the START-M open trial)," Neuroscience and Behavioral Physiology, vol. 38, no. 9, pp. 933-936, 2008.

[29] M. Jog, M. Panisset, O. Suchowersky, B. Réhel, and R. Schecter, "Naturalistic evaluation of entacapone in patients with signs and symptoms of L-dopa wearing-off," Current Medical Research and Opinion, vol. 24, no. 11, pp. 3207-3215, 2008.

[30] W. Ondo, K. Sethi, and G. Kricorian, "Selegiline orally disintegrating tablets in patients with Parkinson disease and "wearing off" symptoms," Clinical Neuropharmacology, vol. 30, no. 5, pp. 295-300, 2007.

[31] G. Deuschl, A. Vaitkus, G. Fox et al., "Efficacy and tolerability of Entacapone versus cabergoline in parkinsonian patients suffering from wearing-off," Movement Disorders, vol. 22, no. 11, pp. 1550-1555, 2007.

[32] D. Brooks, Y. Agid, K. Eggert et al., "Treatment of end-of-dose wearing-off in Parkinson's disease: stalevo (levodopa/carbidopa/entacapone) and levodopa/DDCI given in combination with comtess/comtan (entacapone) provide equivalent improvements in symptom control superior to that of traditional levodopa/DDCI treatment," European Neurology, vol. 53, no. 4, pp. 197-202, 2005.

[33] T. Kondo and Y. Mizuno, "A long-term study of istradefylline safety and efficacy in patients with Parkinson disease," Clinical Neuropharmacology, vol. 38, no. 2, pp. 41-46, 2015.

[34] H. Hao, M. Shao, J. An et al., "Association of catechol-Omethyltransferase and monoamine oxidase $\mathrm{B}$ gene polymorphisms with motor complications in Parkinson's disease in a Chinese population," Parkinson \& Related disorders, vol. 20, no. 10, pp. 1041-1045, 2014.

[35] W. F. Kum, J. Gao, S. S. Durairajan et al., "Risk factors in development of motor complications in Chinese patients with idiopathic Parkinson's disease," Journal of Clinical Neuroscience, vol. 16, no. 8, pp. 1034-1037, 2009.

[36] Q. Sun, T. Wang, T. F. Jiang et al., "Effect of a leucine-rich repeat kinase 2 variant on motor and non-motor symptoms in Chinese Parkinson's disease patients," Aging and Disease, vol. 7, no. 3, pp. 230-236, 2016.

[37] L. M. Melo, H. F. Chien, and E. R. Barbosa, "Identification of wearing-off manifestations (reduction of levodopa effect) in Parkinson's disease using specific questionnaire and comparison of the results with routine ambulatory evaluations," Arquivos de Neuropsiquiatria, vol. 68, no. 4, pp. 506-510, 2010.

[38] M. A. Stacy, H. Murck, and K. Kroenke, "Responsiveness of motor and nonmotor symptoms of Parkinson disease to dopaminergic therapy," Progress in Neuro-Psychopharmacology \& Biological Psychiatry, vol. 34, no. 1, pp. 57-61, 2010.

[39] D. Colombo, G. Abbruzzese, A. Antonini et al., "The "gender factor" in wearing-off among patients with Parkinson's disease: a post hoc analysis of DEEP study," Scientific World Journal, vol. 2015, Article ID 787451, 10 pages, 2015.

[40] P. Martínez-Martín, E. Tolosa, B. Hernández, and X. Badia, "The patient card questionnaire to identify wearing-off in Parkinson disease," Clinical Neuropharmacology, vol. 30, no. 5, pp. 266-275, 2007.

[41] M. C. Altavista, E. Cassetta, L. Brusa et al., "Wearing-off detection in clinical practice: the wearing off real practice key (WORK-PD) study in Parkinson's disease," Parkinsonism Related Disorders, vol. 21, no. 2, pp. 95-100, 2015.

[42] P. A. Silburn, G. D. Mellick, B. I. Vieira et al., "Utility of a patient survey in identifying fluctuations in early stage Parkinson's disease," Journal of Clinical Neuroscience, vol. 15, no. 11, pp. 1235-1239, 2008.
[43] W. Chen, Q. Xiao, M. Shao et al., "Prevalence of wearing-off and dyskinesia among the patients with Parkinson's disease on levodopa therapy: a multi-center registry survey in mainland China," Translational Neurodegeneration, vol. 3, no. 1, pp. 1-8, 2014.

[44] H. Chen, J. Fang, F. Li, L. Gao, and T. Feng, "Risk factors and safe dosage of levodopa for wearing-off phenomenon in Chinese patients with Parkinson's disease," Neurological Sciences, vol. 36, no. 7, pp. 1217-1223, 2015.

[45] M. Picillo, R. Palladino, M. Moccia et al., "Gender and non motor fluctuations in Parkinson's disease: a prospective study," Parkinsonism and Related Disorder, vol. 27, pp. 89-92, 2016.

[46] M. Pistacchi, M. Gioulis, F. Sanson, and S. Marsala, "Wearing off: a complex phenomenon often poorly recognized in Parkinson's disease. A study with the WOQ-19 questionnaire," Neurology India, vol. 65, no. 6, pp. 1271-1279, 2017.

[47] H. Wu, F. Dong, Y. Wang et al., "Catechol-O-methyltransferase Val158Met polymorphism: modulation of wearing-off susceptibility in a Chinese cohort of Parkinson's disease," Parkinsonism and Related Disorder, vol. 20, no. 10, pp. 1094-1096, 2014.

[48] K. E. Rahne, C. Tagesson, and D. Nyholm, "Motor fluctuations and Helicobacter pylori in Parkinson's disease," Journal of Neurology, vol. 260, no. 12, pp. 2974-2980, 2013.

[49] L. Raciti, A. Nicoletti, G. Mostile et al., "Validation of the UPDRS section IV for detection of motor fluctuations in Parkinson's disease," Parkinsonism and Related Disorder, vol. 27, pp. 98-101, 2016.

[50] C. B. Terwee, S. D. Bot, M. R. de Boer et al., "Quality criteria were proposed for measurement properties of health status questionnaires," Journal of Clinical Epidemiology, vol. 60, no. 1, pp. 34-42, 2007. 


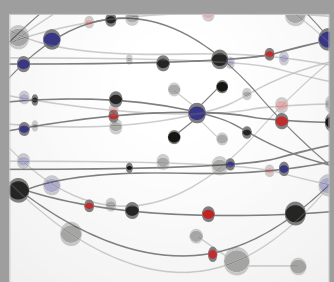

The Scientific World Journal
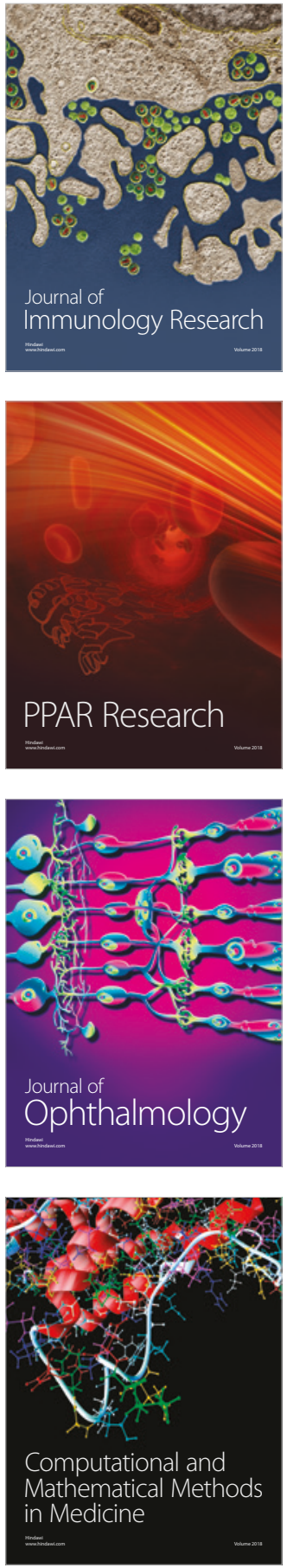

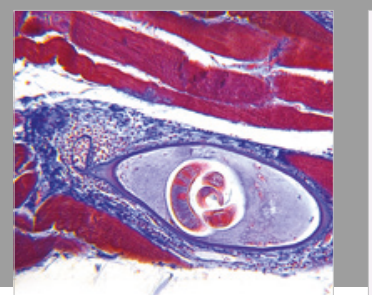

Gastroenterology Research and Practice

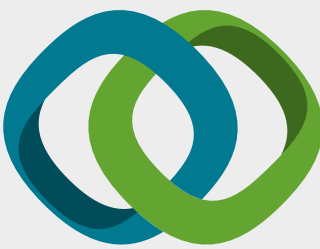

\section{Hindawi}

Submit your manuscripts at

www.hindawi.com
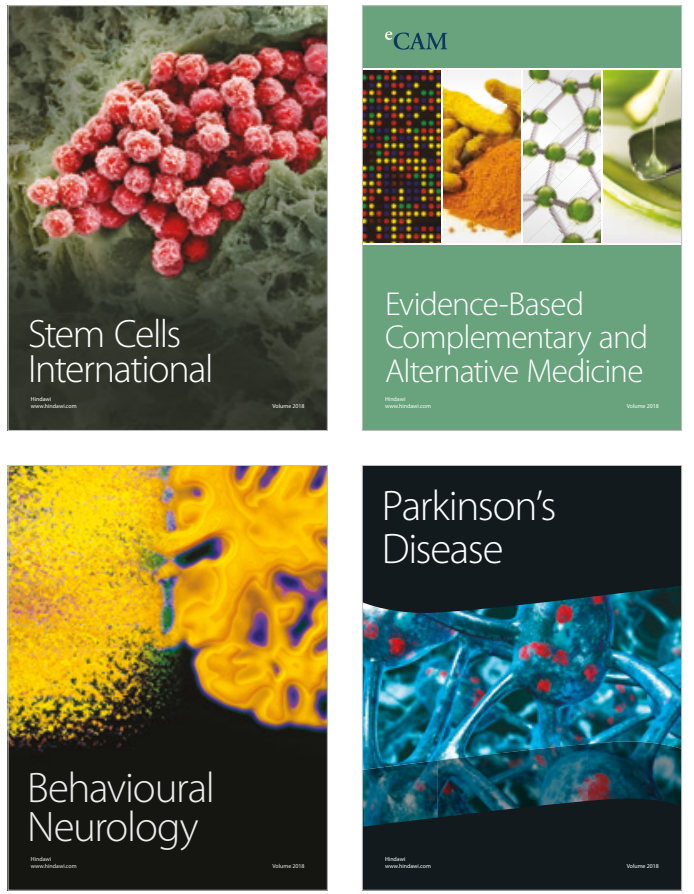



ournal of

Diabetes Research



Disease Markers
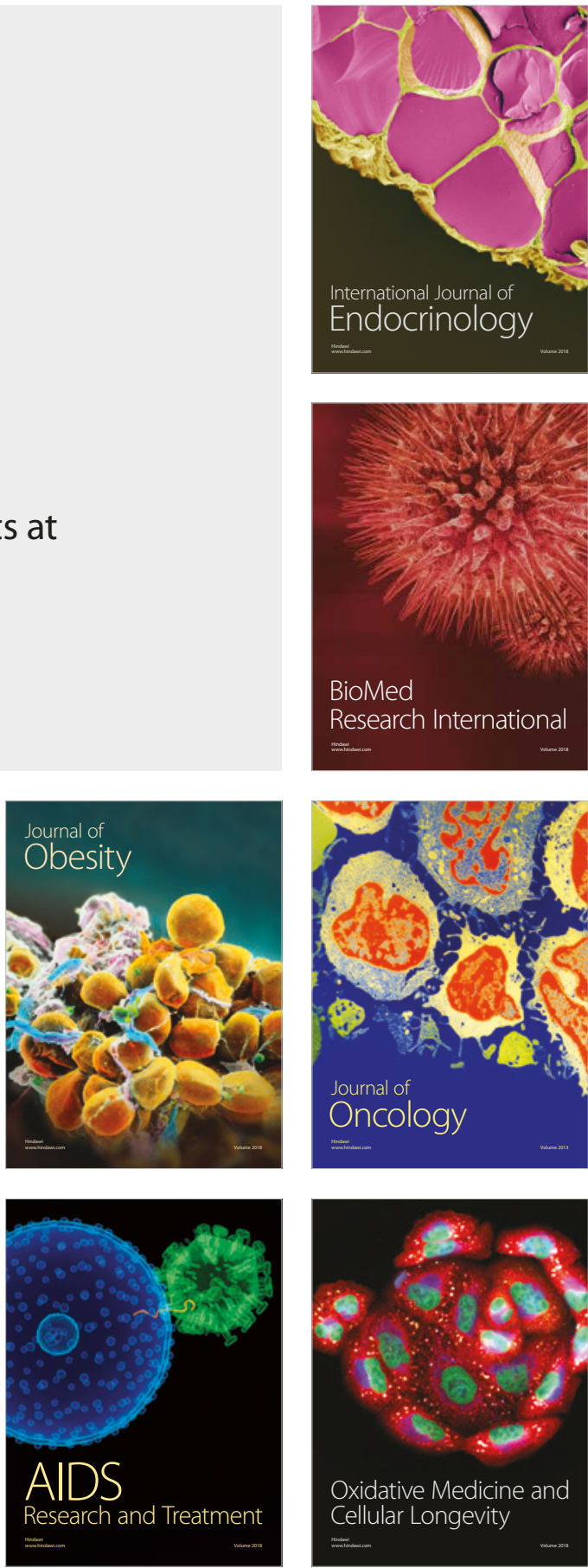